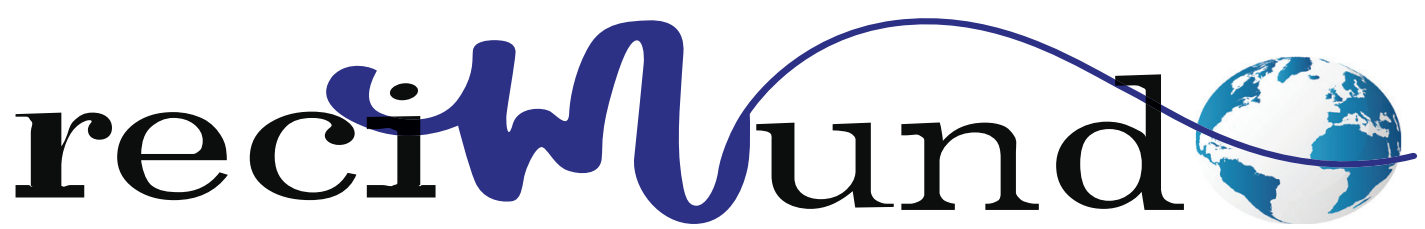

Revista Científica Mundo de la Investigación y el Conocimiento

DOI: 10.26820/recimundo/5.(1).enero.2021.141-152

URL: http://recimundo.com/index.php/es/article/view/993

EDITORIAL: Saberes del Conocimiento

REVISTA: RECIMUNDO

ISSN: 2588-073X

TIPO DE INVESTIGACIÓN: Artículo de Investigación CÓdIGO UNESCO: 1203 Ciencia de Los Ordenadores PAGINAS: $141-152$

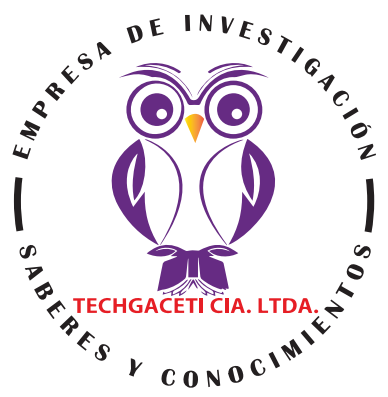

\title{
Inteligencia artificial enfocada al uso y distribución de terrenos para procesos de producción agrícola
}

Artificial intelligence focused on the use and distribution of land for agricultural production processes

Inteligência artificial focalizada no uso e distribuição de terras para processos de produção agrícola

Marjorie Cristina Caicedo Coello; Edwin José Chicaiza Guerra²; Manuel William Villa Quishpe ${ }^{3}$

RECIBIDO: 28/11/2020 ACEPTADO: 06/12/2020 PUBLICADO: 31/01/2021

1. Estudiante de la Facultad ciencias de la ingeniería y aplicadas; Universidad Técnica de Cotopaxi; Latacunga, Cotopaxi; Ecuador; marjorie.caicedo5714@utc.edu.ec; https://orcid.org/0000-0002-7477-9454

2. Estudiante de la Facultad ciencias de la ingeniería y aplicadas; Universidad Técnica de Cotopaxi; Latacunga, Cotopaxi; Ecuador; edwin.chicaiza7877@utc.edu.ec; https://orcid.org/0000-0002-6898-5665

3. Docente de Facultad ciencias de la ingeniería y aplicadas; Universidad Técnica de Cotopaxi; Latacunga, Cotopaxi; Ecuador; manuel.villa@utc.edu.ec; https://orcid.org/0000-0001-5827-8337

CORRESPONDENCIA

Caicedo Coello Marjorie Cristina

marjorie.caicedo5714@utc.edu.ec

Latacunga, Ecuador

○ RECIMUNDO; Editorial Saberes del Conocimiento, 2021 


\section{RESUMEN}

La comunidad agrícola siendo una de las fuentes más económicas e importantes del Ecuador ha presentado varias necesidades al momento de optimizar recursos en el proceso de cultivo. Una de las necesidades que ha presentado la comunidad agricultora es el encontrar una solución efectiva ante la falta de conocimiento sobre la medición de su terreno para la construcción de invernaderos y el estado del suelo permitiendo conocer la cantidad exacta de plantas a ser sembradas, aportando un mayor grado de efectividad y satisfacción a la comunidad agrícola, que trabaja bajo invernadero. En el presente documento se explica acerca del desarrolló de un módulo de medición de terreno aplicando técnicas de inteligencia artificial, las cuales ayudaron con las necesidades que tienen los agricultores de la comunidad. El módulo mencionado facilitó al personal encargado en el procedimiento de la medición del terreno, y al efectuar el cálculo de número de plantas que entran en las camas del invernadero. Para el desarrollo de este módulo se utilizó la metodología de investigación mixta ya que se realizó un análisis cuantitativo-cualitativo, y a su vez una metodología de desarrollo ágil como Mobile D. Se tomaron en cuenta varias técnicas de la inteligencia artificial para optimizar tiempo y recursos en sectores agrícolas. Al analizar y aplicar estas técnicas hemos podido constatar el estado del terreno bajo invernadero permitiendo realizar la medición correcta con la ayuda de GoogleMaps a través de la utilización de coordenadas UTM que permite extraer el ancho y longitud del terreno bajo invernadero.

Palabras clave: Complemento, control, datos, módulo, móvil.

\section{ABSTRACT}

The agricultural community, being one of the most economical and important sources in Ecuador, has presented several needs when optimizing resources in the cultivation process. One of the needs presented by the farming community is to find an effective solution to the lack of knowledge about the measurement of their land for the construction of greenhouses and the state of the soil, allowing them to know the exact number of plants to be planted, providing a greater degree of effectiveness and satisfaction to the agricultural community, which works under the greenhouse. This document explains the development of a field measurement module applying artificial intelligence techniques, which helped with the needs of community farmers. The mentioned module facilitated the personnel in charge in the procedure of the measurement of the land, and when carrying out the calculation of the number of plants that enters the beds of the greenhouse. For the development of this module, the mixed research methodology was used since a quantitative-qualitative analysis was carried out, and in turn, an agile development methodology such as Mo-bile D. Various techniques of artificial intelligence was taken into account to optimize time and resources in agricultural sectors. By analyzing and applying these techniques we have been able to verify the state of the land under the greenhouse, allowing us to make the correct measurement with the help of Google Maps through the use of UTM coordinates that allow us to extract the width and length of the land under the greenhouse.

Keywords: Complement, control, data, module, mobile.

\section{RESUMO}

A comunidade agrícola, sendo uma das fontes mais econômicas e importantes do Equador, apresentou várias necessidades ao otimizar os recursos no processo de cultivo. Uma das necessidades apresentadas pela comunidade agrícola é encontrar uma solução eficaz para a falta de conhecimento sobre a medição de suas terras para a construção de estufas e o estado do solo, permitindo-lhes conhecer o número exato de plantas a serem plantadas, proporcionando um maior grau de eficácia e satisfação à comunidade agrícola, que trabalha sob a estufa. Este documento explica o desenvolvimento de um módulo de medição de campo aplicando técnicas de inteligência artificial, o que ajudou a atender as necessidades dos agricultores comunitários. O referido módulo facilitou o pessoal encarregado no procedimento de medição do terreno e no cálculo do número de plantas que entram nos canteiros da estufa. Para o desenvolvimento deste módulo, foi utilizada a metodologia de pesquisa mista, uma vez que foi realizada uma análise quantitativa-qualitativa e, por sua vez, uma metodologia ágil de desenvolvimento como a Mo-bile D. Várias técnicas de inteligência artificial foram levadas em conta para otimizar o tempo e os recursos nos setores agrícolas. Analisando e aplicando estas técnicas, pudemos verificar o estado do terreno sob a estufa, permitindo-nos fazer a medida correta com a ajuda do Google Maps através do uso de coordenadas UTM que nos permitem extrair a largura e o comprimento do terreno sob a estufa.

Palavras-chave: Complemento, controle, dados, módulo, móvel. 


\section{Introducción}

Alrededor del planeta según el Foro Rural mundial aproximadamente más de 3,000 millones de personas viven en el campo. Y de estos unos 2,500 millones, son agricultores correspondiente a hombres y mujeres donde aproximadamente 1,500 millones posen parcelas menores de 2 hectáreas o menor a una hectárea, un dato importante es que los Países Bajos tienen una posición dominante en la horticultura a nivel mundial [1]. Este país tiene una gran especialización en la construcción y manejo de invernaderos ya que cuenta con más de 10.000 hectáreas, donde el mercado de recambio tiene prioridad. El invernadero de cristal es el artículo de exportación neerlandés por excelencia. Ochenta por ciento de los invernaderos de cristal fuera de Europa proviene de los Países Bajos [2].

Adicional a lo anterior podemos mencionar que según el censo agropecuario en 2008 en EL Salvador se registra 390.475 unidades productivas, aproximadamente 3 hectáreas o el $85.8 \%$, pero estas según el Ministerios de Agricultura del El Salvador, proveen más del $70 \%$ de la producción agrícola básica. Esto hace que estas unidades productivas se conviertan en una parte fundamental e importante para los seres humanos ya que forman parte de lo indispensable para su propia supervivencia y desarrollo de las zonas rurales y urbanas en El Salvador se caracteriza por ser el máximo exportador y proveedor de productos agrícolas, convirtiéndose así en las principales proveedoras de los alimentos básicos que tiene en el país como es el maíz y frijol [1].

En el sector agrícola del Ecuador en la región sierra, la topografía del terreno presenta desniveles importantes en su extensión. El clima de esta zona es variado, muchos de estos sectores son minifundistas, se dedican al cultivo de sus pequeñas propiedades, los productos más conocidos en la zona son: cabuya, maíz, cebada, trigo y otros cereales de clima templado que soportan la sequía, por tratarse de terrenos arenosos y sin agua de regadío.

Uno de los principales problemas que afecta al sector agrícola es el desperdicio de recursos agrícolas ya que no poseen una herramienta que les permita calcular los recursos exactos para la siembra y construcción de invernadero. Dado a esto se pensó en algunas ideas que sean suficientemente capaces de ayudar a solucionar este tipo de problemas, es ahí donde aparece la automatización de invernaderos, la cual no solo se encarga de realizar la medición del invernadero; sino que también les dará la cantidad y la posición exacta de cada cultivo, para que posteriormente sea plantada en el invernadero.

La autoría del presente documento es de estudiantes y docentes de la carrera de Ingeniería en Informática y Sistemas Computacionales de la Universidad Técnica de Cotopaxi (UTC), quienes con el auspicio de la Universidad Técnica de Cotopaxi han delineado el desarrollo del proyecto de investigación Técnicas de inteligencia artificial para la producción agrícola en la provincia de Cotopaxi. A través de la aplicación de Mobile D como metodología de desarrollo para el módulo de medición de terreno aplicando técnicas de inteligencia artificial, donde a través de la utilización de varios métodos como del círculo, cuadrado, rectángulo para obtener el sistema de coordenadas UTM que permite extraer el ancho y longitud del terreno bajo invernadero.

El resto del documento describe el desarrollo y posterior análisis de resultados donde se explica brevemente acerca del desarroIlo, cálculo de dimensiones como de cantidades de surcos y plantas. Finalmente se incluyen las conclusiones del trabajo. 


\section{Metodología}

\section{Métodos para cálculo de dimensiones en el terreno}

Para el cálculo de las dimensiones se ha utilizado GoogleMaps como complemento. Esta herramienta utiliza varios métodos entre estos tenemos el método del círculo, el método del polígono (suma del área del triángulo), método del cuadrado y método de aproximación.

En este módulo se utiliza el método rectangular sin considerar el contorno de la tierra donde se aplica la determinación de las dimensiones de un invernadero. Este método toma en cuenta un punto en la superficie con coordenadas sea esta latitud y longitud Ec. (1) para su posterior cálculo o transformación en el sistema de coordenadas UTM como lo muestra la siguiente imagen [3].

$$
\begin{aligned}
& e^{e}=e^{e} /\left(1-e^{2}\right) \\
& \mathrm{N}=a /\left(1-e^{2} \sin ^{2}(\varnothing)\right)^{1 / 2} \\
& \mathrm{~T}=\tan ^{2} \emptyset \\
& \mathrm{C}=e^{\prime 2} \cos ^{2}(\varnothing) \\
& \mathrm{A}=\left(\gamma-\gamma_{0}\right) \cos (\emptyset) \\
& \mathrm{M}=\left[\begin{array}{c}
\left(1-\frac{e^{2}}{4}-\frac{3 e^{4}}{64}-\frac{5 e^{6}}{256}-\cdots\right) \emptyset-\left(\frac{3 e^{2}}{8}+\frac{3 e^{3}}{32}+\frac{45 e^{6}}{1024}+\cdots\right) \sin (2 \emptyset) \\
+\left(\frac{15 e^{4}}{256}+\frac{45 e^{6}}{1024}+\cdots\right) \sin (4 \varnothing)-\left(\frac{15 e^{6}}{3072}+\cdots\right) \sin (6 \varnothing)+\cdots
\end{array}\right]
\end{aligned}
$$

Tal que el Este se puede encontrar usando la fórmula Ec. (2)

$$
\mathrm{x}=k_{0} N\left[A+(1-T+C) \frac{A^{3}}{6}+\left(5-18 T+T^{2}+72 C-58 e^{\prime 2}\right) \frac{A^{5}}{120}\right],
$$

Y el norte se puede encontrar usando la fórmula Ec. (3)

$$
\mathrm{y}=k_{0}\left[M-M_{0}+N \tan (\varnothing)\left(\frac{A^{2}}{2}+\left(5-T+9 C+4 C^{2}\right) \frac{A^{4}}{24}+B \frac{A^{6}}{720}\right)\right]
$$

\section{Método para el cálculo de cantidad de plantas y surcos}

La necesidad de crear mecanismos autónomos capaces de evaluar las características visuales de una escena en el campo de cultivo y tomar decisiones a partir del conjunto de información que recogen es cada vez mayor [4]. Es por esto que la automatización de los procesos agrícolas es importante donde los procesos agrícolas utilizan sis- temas de visión por computador, instalados a bordo de vehículos agrícolas, está ganando una relevancia importante hoy en día.

Regresión lineal: para el ajuste de polinomios (lineal o cuadrático) que definen las líneas de cultivo donde se obtienen resultados aceptables en precisión como en el tiempo de procesamiento. Este método el favorable para terrenos con baja densidad de malas hierbas. Ya que Este enfoque es- 
tima la pendiente y el intercepto de la ecuación de la línea recta y asigna una ecuación a cada una de las líneas de cultivo.

\section{Mobile-D}

Para el desarrollo de la aplicación móvil se utilizó la metodología Mobile-D, marco de trabajo específico para implementar soluciones de este tipo.

\section{Fases de la metodología Mobile-D:}

Las etapas del ciclo de desarrollo de la metodología Mobile-D son:

- Exploración: Donde se define el alcance del proyecto y la especificación de funcionalidades.

- Inicialización: Se realiza la configuración del entorno de programación; es decir, se preparan todos los recursos y herramientas necesarias para el inicio de la fase de producción.

- Producción: En esta fase se realiza la implementación de la solución.

- Estabilización: Se emprende la etapa de aseguramiento de la calidad de la solución implementada; donde se verifican los procesos de desarrollo en cada fase.

- Pruebas del sistema: En esta fase se comprueba si el producto implementa correctamente las funcionalidades requeridas; es decir, se realizan las pruebas necesarias para garantizar un despliegue sin errores [5].

\section{Desarrollo}

\section{Inteligencia artificial}

Es un campo de la ciencia y de la ingeniería que se ocupa de la comprensión a través de la computadora de lo que comúnmente llamamos comportamiento inteligente y de la creación de herramientas que exhiben tal comportamiento [6].

\section{Técnicas de inteligencia artificial}

\section{Sistemas expertos}

Según Valverde menciona que "Los sistemas expertos se constituyen en la herramienta de la Inteligencia artificial más utilizada desde sus inicios" ya que estos son aquellos programas de ordenador o de dispositivos móviles que recopilan, procesan y analizan el conocimiento de especialistas en un software [7].

Como base conocimiento en este módulo se ha definido a los docentes y estudiantes de la Facultad de CAREN de la Universidad Técnica de Cotopaxi, ya que proveen de conocimiento, experiencia en el sector agrícola.

Como motor de inferencia se ha utilizado el entorno de desarrollo Android studio y GoogleMaps que nos permite obtener conclusiones de la base de conocimiento mediante el procesamiento adecuado de la información empleando lenguajes de programación.

Como primer paso se ha realizado entrevistas a expertos en el sector agropecuario para recopilarlas, seleccionarlas y retroalimentar nuestra base de conocimiento en nuestro caso una base de datos con replicación circular.A continuación se ha diseñado reglas de inferencia que manejara el módulo para ser experto con bases de entradas proporcionadas por el usuario a través de una interfaz.

Este módulo al interactuar constantemente con el usuario y la base de conocimiento podrá , realizar el cálculo de las dimensiones del terreno bajo invernadero así como el cálculo de surcos y número exacto de camas, con la aplicación de varios métodos ya mencionados. A continuación, se detalla el proceso realizado para construir el módulo. 
Aplicación móvil Requerimientos funcionales

Para el módulo de medición de terreno se definieron dos requisitos funcionales.

- RF01: Cálculo de dimensiones.

- RF02: Cálculo de cantidades.

De acuerdo con los expertos en el tema, los requisitos de gran importancia en este módulo se describen en la tabla 1 y 2.

Tabla 1. Requerimiento funcional cálculo de dimensiones

\begin{tabular}{|c|c|}
\hline$N^{\mathbf{0}}$ : & RF01 \\
\hline $\begin{array}{c}\text { Nombre del } \\
\text { requisito: }\end{array}$ & Cálculo de dimensiones \\
\hline Prioridad: & Alta \\
\hline Datos de Entrada: & Longitud, anchura \\
\hline $\begin{array}{c}\text { Proceso ingresar: } \\
\text { Requerimiento no } \\
\text { funcional: }\end{array}$ & $\begin{array}{c}\text { Se calcula el área del } \\
\text { terreno }\end{array}$ \\
\hline Datos de salida: & Área de terreno \\
05
\end{tabular}

Tabla 2. Cálculo de cantidades

\begin{tabular}{|c|c|}
\hline $\mathbf{N}^{\mathbf{0}}$ : & RF02 \\
\hline $\begin{array}{c}\text { Nombre del } \\
\text { requisito: }\end{array}$ & Cálculo de cantidades \\
\hline Prioridad: & Alta \\
\hline Datos de Entrada: & Área del terreno \\
\hline $\begin{array}{c}\text { Proceso ingresar: } \\
\text { Requerimiento no } \\
\text { funcional: }\end{array}$ & $\begin{array}{c}\text { Se calcula el número de } \\
\text { plantas que el usuario } \\
\text { elija. }\end{array}$ \\
\hline $\begin{array}{c}\text { Datos de salida: } \\
\text { RNF-02,RN-03, RNF- } \\
05\end{array}$ \\
\hline
\end{tabular}

\section{Requerimientos no funcionales}

- RNF-01 - Interfaz del sistema: La interfaz del módulo debe estar implementada en la aplicación móvil.

- RNF-02 - Usabilidad: el módulo deberá proveer una interfaz amigable con el usuario, fácil de aprender y navegar.

- RNF-03 - Portabilidad: El módulo siem- pre deberá estar conectado a la replicación del servidor.

- RNF-04 - Rapidez: El módulo deberá tener un tiempo máximo de respuesta entre 2 y 4 segundos en el servidor de replicación.

- RNF-05 - Operatividad: El sistema debe visualizarse y funcionar correctamente en el dispositivo.

\section{Configuración del ambiente de desarrollo}

Se configuraron las siguientes herramientas de desarrollo:

Para el desarrollo del módulo se configuraron las siguientes herramientas:

Base de datos: MySQL como gestor de base de datos que se basa en lenguaje de consulta estructurado, juntamente con Xamp donde se realizará la conexión maestro-esclavo.

\section{Web service: Framework JSON}

Para el funcionamiento de la aplicación y la replicación de los datos Android necesita una API intermedia que le permita interactuar con el servidor de base de datos. Esta capa se llama JSON que permite enviar y recibir peticiones a través del protocolo HTPP.

\section{Configuración de la aplicación móvil}

Entorno de desarrollo: Android Studio

\section{Fase de producción}

En esta fase se implementan todos los requisitos que necesita el módulo en las diferentes iteraciones donde se ejecutaron procesos de análisis, diseño, codificación y pruebas; la figura 1 describe el modelo de casos de uso general. 


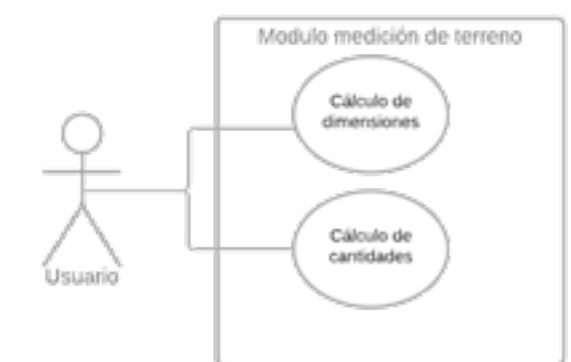

Figura 1. Caso de uso general de aplicación móvil.

Una vez que se definieron los requisitos, se procede a diseñar el prototipo de las interfaces gráficas de usuario (IGU), para ello se utilizó la herramienta informática.

AppMoqups. La figura 2 permite realizar la medición del terreno con Google Maps.

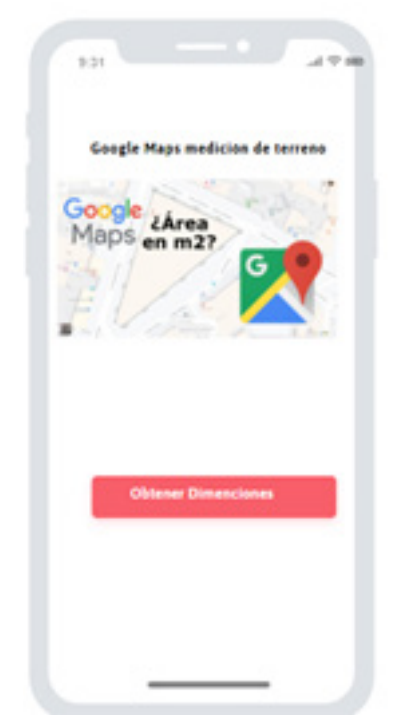

Figura 2. Mockup cálculo del dimensión del terreno

La herramienta más adecuada que nos permite realizar la medición correcta es GoogleMaps como complemento del módulo, donde se obtiene la información del terreno bajo invernadero a través de la utilización de varios métodos como del círculo, cuadrado, rectángulo para obtener el sistema de coordenadas UTM que permite extraer el ancho y longitud del terreno bajo invernadero.

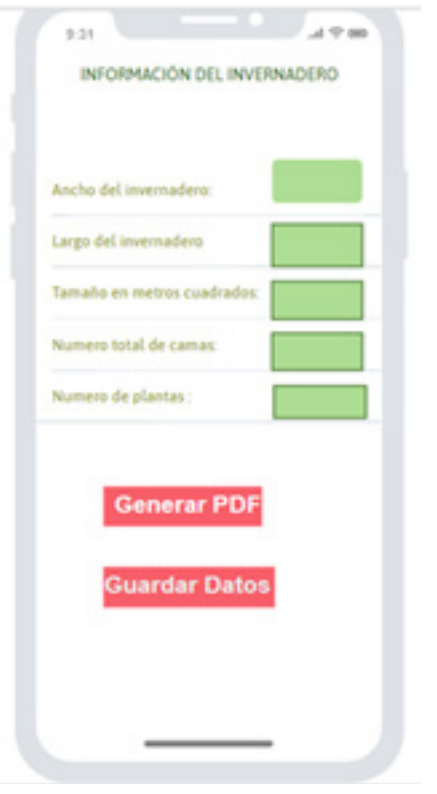

Figura 3. Mockup Cálculo de cantidades.

Los datos obtenidos por la herramienta de complemento del módulo son visualizados y tratados (Dirección del invernadero, él ancho y largo del invernadero) para su posterior cálculo para cumplir al máximo su funcionalidad.

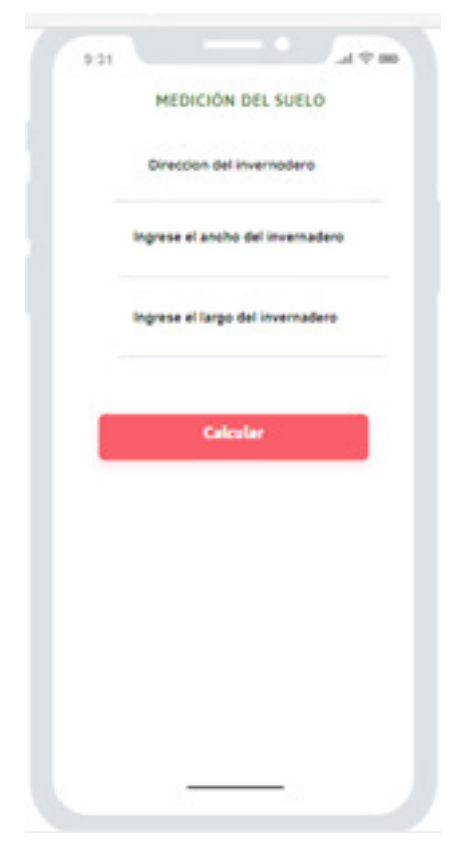

Figura 4. Mockup Procesamiento de datos 
Una vez calculado todo se visualiza la información recolectada por la herramienta móvil, la cual permite definir la cantidad exacta de plantas que se pueden sembrar en todo el terreno y de la misma manera sucede con los surcos o camas, ayudando a los agricultores a generar pdf como reportes y de la misma forma guardar las medidas para un futuro uso.

Como siguiente paso se realizaron los diagramas estructurales y dinámicos de la aplicación, se diseñaron los diagramas de clases, de secuencias, de actividades, entre otros; en la figura 5 se muestra el modelo de la base de datos.

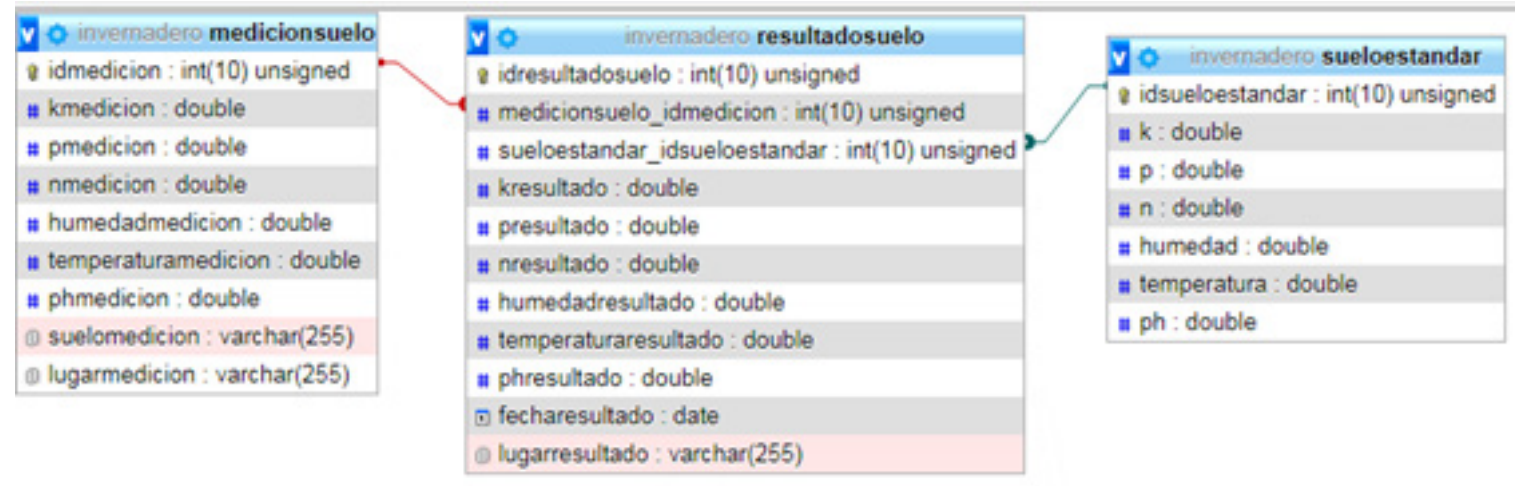

Figura 5. Modelo de la base de datos del módulo

Una vez desarrollada la base de datos con sus respectivas relaciones se realizó el código en Php que servirá de intermediario de conexión entre el módulo y la base de datos, así mismo de para almacenar, replicar datos tal como se puede observar en la figura 6 y la figura 7.

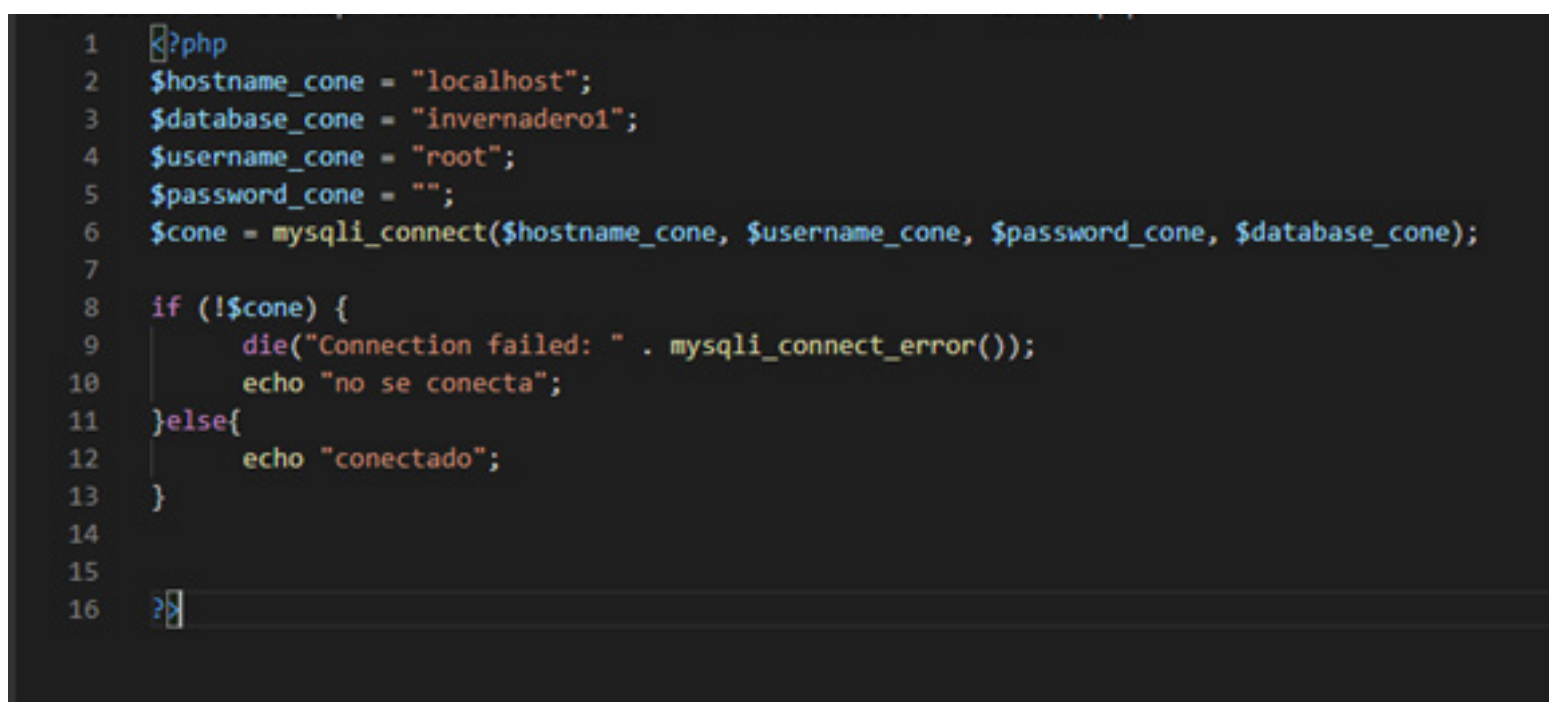

Figura 6. Desarrollo del código en Php de conexión ente el modulo y la base de datos 


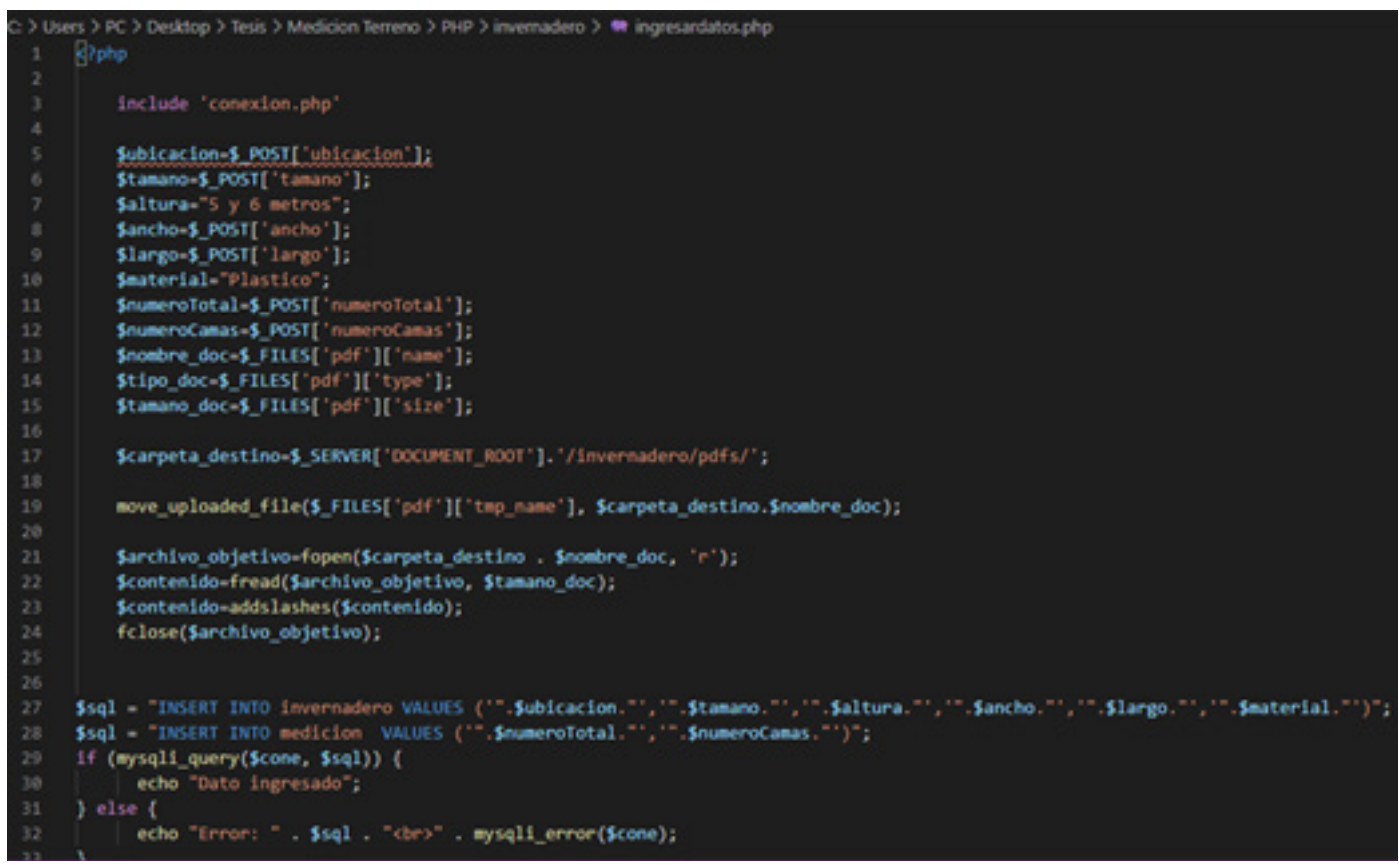

Figura 7. Desarrollo del código en php para guardar los datos de la medición del terreno

Luego de crear los respectivos archivos intermediarios procedemos a llamarlo desde el entorno de programación que se realizó el modulo tal y como se puede observar en la figura. Para poder sacar el máximo del provecho al módulo.

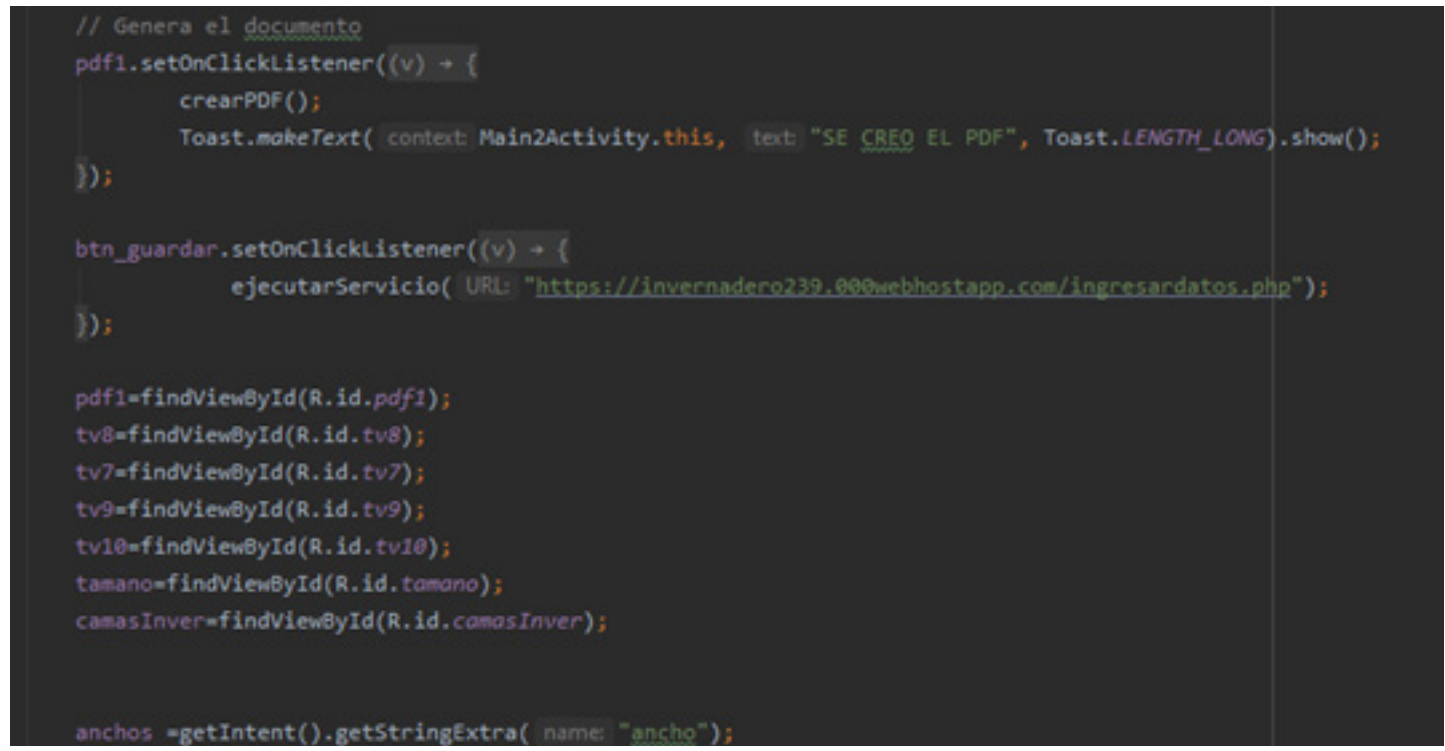

Figura 8. En la línea de ejecutar servicio se realiza el llamado al código intermediario de conexión

Después de preparar a la aplicación se da su funcionamiento en donde primero realizamos la medición del terreno desde un punto a otro sacando las medidas de largo y ancho datos necesarios para realizar la construcción y el cálculo de los productos agrícolas hacer sembrados con sus respectivas dimensiones como se puede observar en la figura 9. 

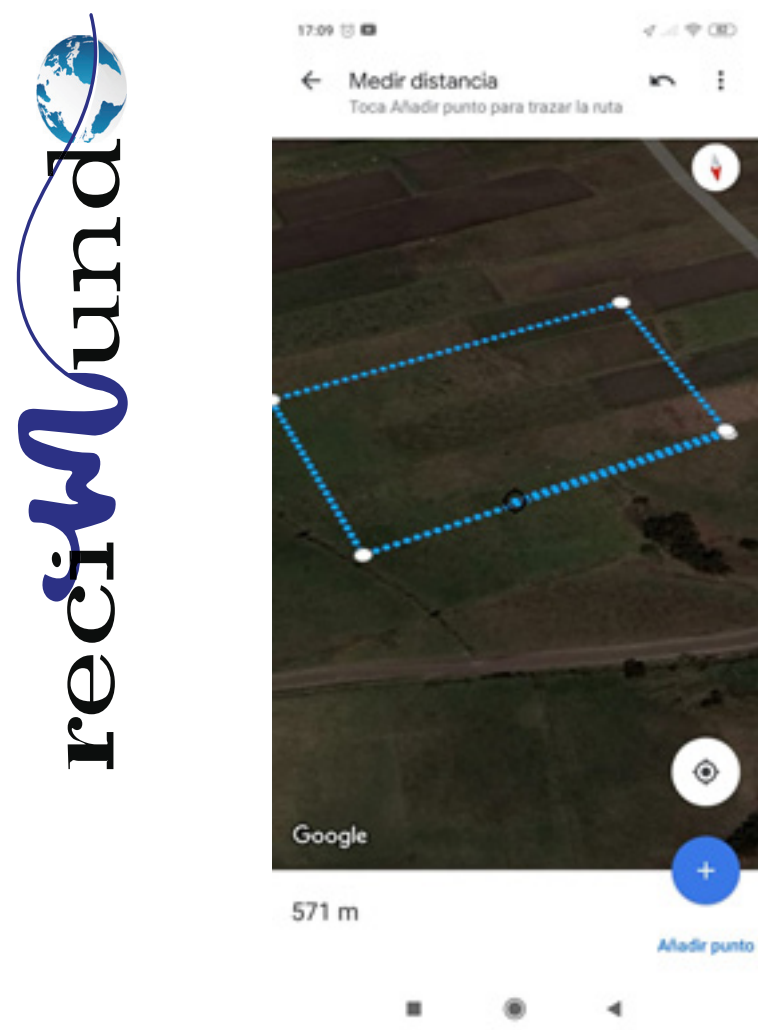

Figura 9. Obtención de la medición del terreno para la construcción del invernadero

Después de realizar los diseños necesarios para definir los procesos, métodos y técnicas que nos permitirán realizar los cálculos sin problema, procedemos a la codificación de cada caso de uso y se procede a hacer pruebas para comprobar su funcionalidad. La figura 10 ilustra la interfaz gráfica para realizar las operaciones del módulo de medición.

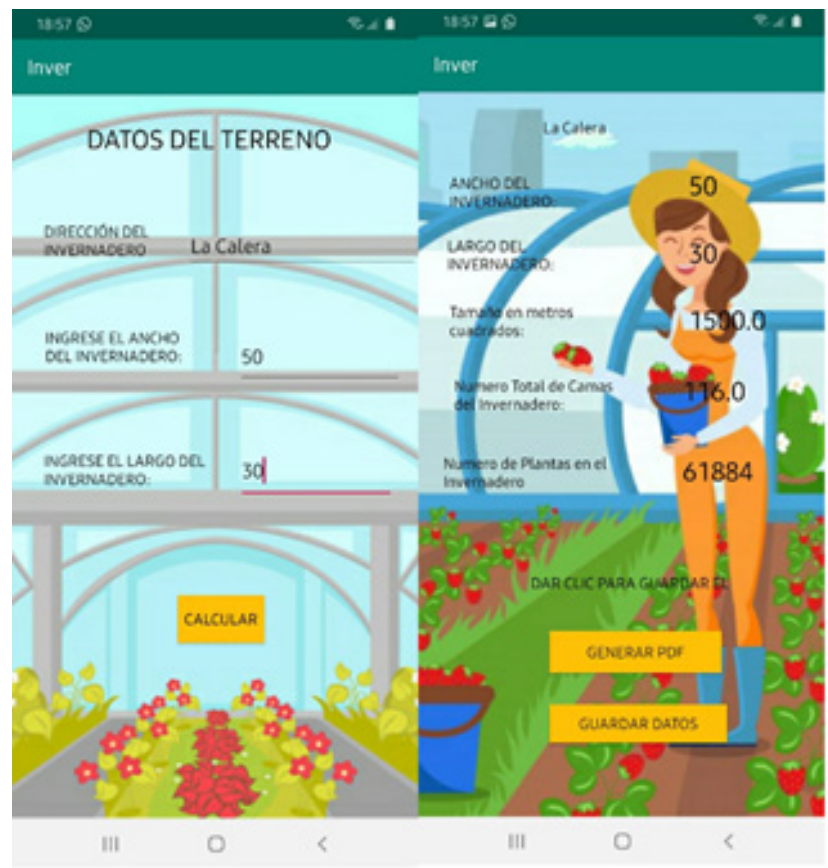

Figura 10. Captura de pantalla cálculo de surcos y plantas

\section{Discusión y resultados}

\section{Aplicación en producción}

El desarrollo del módulo y la aplicación móvil presenta proporciona al usuario una interfaz amigable, para el cálculo de cantidades exactas de surcos y plantas como se visualiza en la figura 10. Ayudando no solo a optimizar recursos sino también a enriquecer los conocimientos de la comunidad agrícola.

Adicional a esto el modulo permite registrar los datos obtenidos y calculados en el gestor de base de datos MySQL como se visualiza en la figura 11. Además se realiza una replicación en círculo facilitando a los usurarios realizar cálculos al mismo tiempo. La aplicación móvil otorga toda la información completa para la comprar de recursos exactos para la siembra como se visualiza en la figura10. 


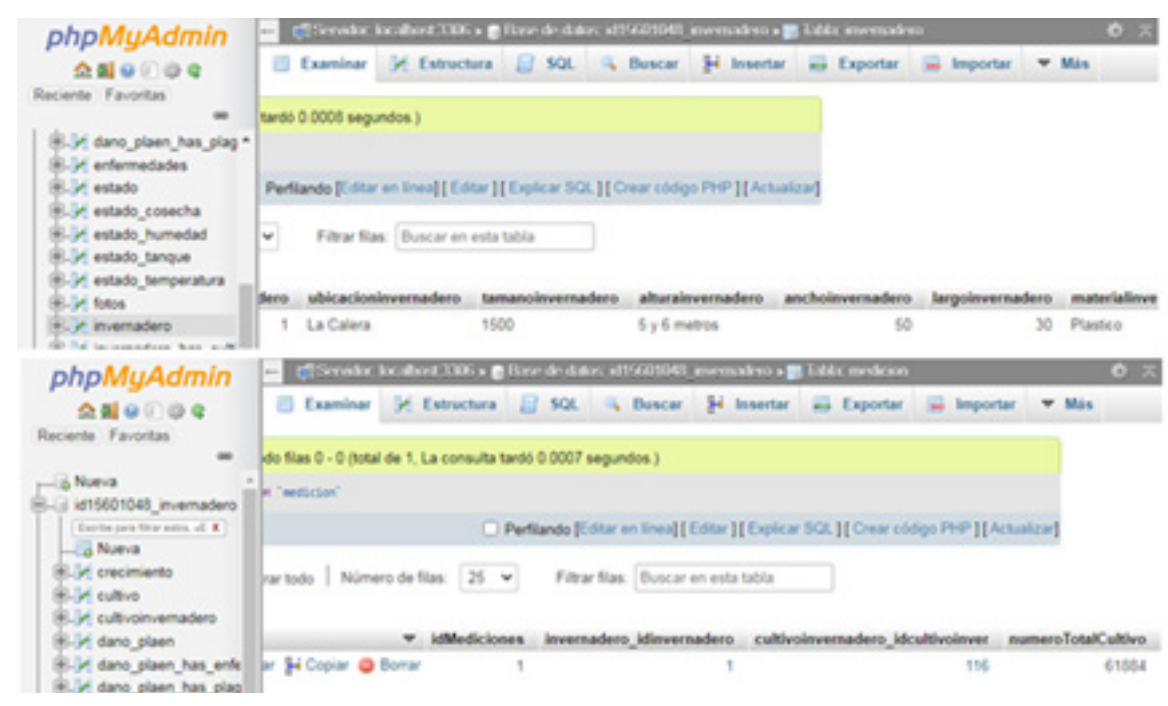

Figura 11. Captura de pantalla de los datos en el servidor

Este módulo juntamente con la aplicación móvil en relación a otras aplicaciones similares como la [8] y la [9] permite al usuario calcular el número exacto de surcos según el tamaño del terreno bajo invernadero.

Razón por la que no se puede discutir su funcionamiento eficaz y certero; sin embargo, se puede mencionar a favor del trabajo que se presenta en este documento, que se analizó, diseño e implemento este módulo en la herramienta Android Studio [10] la que permite diseñar y desarrollar aplicaciones para dispositivos móviles con sistema operativo Android.

\section{Conclusiones}

El uso de inteligencia artificial así como de sus técnicas en diferentes campos actualmente es muy popular, ya que facilitan el trabajo del ser humano optimizando tiempo, recursos económicos. Una de las técnicas más relevantes que presenta la inteligencia artificial con los sistemas expertos ya que se crean sistemas autónomos, donde se lo alimenta con conocimiento constantemente. Los objetivos plasmados en el desarrollo de esta investigación y posterior desarrollo del módulo, se ha conseguido el cálculo del número exacto de surcos y plantas para la op- timización de recursos en el sector agrícola. El desarrollo de este módulo ha sido de gran importancia y necesidad por parte del sector agrícola, por ello cuenta con gran aceptación por parte de este y a la vez como complemento al sistema de monitoreo para la producción agrícola.

La inteligencia artificial esta inmiscuida en el sector agrícola con el fin de beneficiar no solo a los agricultores y a su vez disminuye la contaminación, cuidando y preservando de los recursos naturales preservando el ecosistema facilitando cálculos precisos de información.

\section{Bibliografía}

[1] E. A. Amaya Garcia and R. A. Y. Walter, "DISEÑO DE AUTOMATIZACIÓN DE SISTEMA DE RIEGO DE INVERNADERO PARA EL DESARROLLO DE LA AGRICULTURA FAMILIAR EN EL MARCO DE LA SEGURIDAD ALIMENTARIA," EI Salvador, 2016.

[2] O. de la N. U. para la Alimentación, "The state of agriculture and food," in El estado Mundial de la Agricultura y la Alimentación, 2017, pp. 1-178, [Online]. Available: http://www.fao.org/3/a-i7658s. pdf.

[3] A. Setiawan and E. Sediyono, "THE USE OF GOOGLE MAPS AND UNIVERSAL TRANSVERSE MERCATOR (UTM) COORDINATE IN LAND MEASUREMENT OF REGION IN DIFFERENT ZONE," J. Theor. Appl. Inf. Technol., vol. 15, no. 23, 2018, Ac-

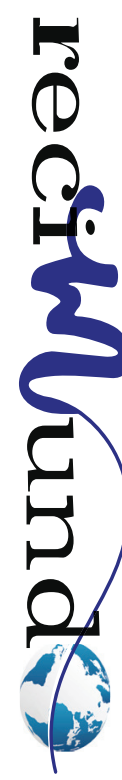


cessed: Dec. 15, 2020. [Online]. Available: www. jatit.org.

[4] I. D. García Santillán, "Métodos de visión por computador para detección automática de líneas de cultivo curvas/rectas y malas hierbas en campos de maíz," UNIVERSIDAD COMPLUTENSE DE MADRID, 2018.

[5] W. A. Zambrano García, "Estudio Comparativo De Metodologías De Desarrollo Ágil En Base Al Desarrollo De Una Aplicación Móvil, Modalidad Proyecto De Investigación, Para La Obtención Del Título De Ingeniero InformáticoEstudio Comparativo De Metodologías De Desarrollo Ágil En Base ," UNIVERSIDAD CENTRAL DEL ECUADOR FACULTAD, 2017.
[6] L. E. S. Inaoe, "Métodos de Inteligencia Artificial."

[7] S. Valverde Bourdié, "APLICACIONES DE LA INTELIGENCIA ARTIFICIAL EN LA EMPRESA," Universidad de Cantabria, 2019.

[8] A. Ramírez, "¿QUÉ ES UN GPS DE PRECISIÓN?" https://www.clubensayos.com/Tecnología/QUÉES-UN-GPS-DE-PRECISIÓN/4243411.html (accessed Nov. 27, 2020).

[9] D. O. Poveda Prieto, "LA TECNIFICACIÓN COMO HERRAMIENTA PARA INCREMENTAR LA PRODUCTIVIDAD AGROPECUARIA EN COLOMBIA," FUNDACIÓN UNIVERSIDAD DE AMÉRICA, 2019.

[10] T. Hagos and T. Hagos, "Android Studio," in Learn Android Studio 3, Apress, 2018, pp. 5-17.

\section{ఆ6) \\ CREATIVE COMMONS RECONOCIMIENTO-NOCO- MERCIAL-COMPARTIRIGUAL 4.0.}

\section{CITAR ESTE ARTICULO:}

Caicedo Coello, M. C., Chicaiza Guerra, E. J., \& Villa Quishpe, M. W. (2021). Inteligencia artificial enfocada al uso y distribución de terrenos para procesos de producción agrícola. RECIMUNDO, 5(1), 141-152. https://doi.org/10.26820/ recimundo/5.(1).enero.2021.141-152 